\title{
Variscita y ámbar en el Neolítico gallego. Análisis arqueométrico del collar del túmulo 1 de Chousa Nova, Silleda (Pontevedra, España)
}

\author{
Variscite and amber in the Galician Neolithic. Archaeometric analysis of the necklace \\ from Barrow 1 at Chousa Nova, Silleda (Pontevedra, Spain)
}

\author{
Salvador Domínguez-Bella $(*)$ \\ María José Bóveda $(* *)$
}

\begin{abstract}
RESUMEN
La excavación del túmulo 1 de Chousa Nova, Silleda (Pontevedra), proporcionó unos resultados sorprendentes. Aunque muy deteriorado, por diferentes factores, conservaba evidencias de una gran complejidad constructiva. Dentro de su cámara megalítica apareció, junto con otros objetos pulidos, un conjunto de 35 cuentas dispuestas a modo de collar. Se han estudiado todas, unas de color verde y otras de carácter orgánico, intercaladas entre ellas. Los resultados del análisis arqueométrico mediante técnicas no-destructivas (difracción y fluorescencia de Rayos X, espectroscopía de infrarrojos, microscopía óptica y electrónica de barrido ambiental, con microanálisis) muestran una naturaleza mineralógica basada en la variscita y el ámbar, así como unas características texturales y composicionales muy similares entre cada conjunto. Se ha determinado y cuantificado asimismo la importancia de los fenómenos de alteración y disolución de estos objetos, debido a un ataque químico intenso durante el período de enterramiento. Se discuten las posibles áreas fuente de procedencia de dichos materiales, en base a los datos geoquímicos obtenidos.
\end{abstract}

\begin{abstract}
The excavation of barrow 1 of Chousa Nova, Silleda, Pontevedra (NW Spain) offered surprising results. This mound, in spite of experiencing a large number and types
\end{abstract}

(*) Dpto. de Ciencias de la Tierra y Unidad de Geoarqueología y Arqueometría Aplicadas al Patrimonio Histórico-Artístico y Monumental. Facultad de Ciencias. Universidad de Cádiz. Puerto Real.11510 Cádiz. Correo e.: salvador.dominguez@uca.es

(**) Gabinete de Arqueoloxía e Xestión do Patrimonio S.L. Vía Edison 19C 2. ${ }^{\circ}$ Polígono do Tambre. 15890 Santiago de Compostela. Correo e.: majogabinete@mundo-r.com

Recibido: 17-XII-2010; aceptado 24-I-2011. of anthropic damage, still preserved a great constructive complexity. Within its megalithic chamber, formed by 5 granite orthostats and a large roofing slab of, also in granite, a set of archaeological materials appeared. These were an axe, a chisel and a polishing stone, with a set of 35 beads, most of them of a green stone and the rest of an organic material, with an extremely high degree of alteration, inserted between them. The analytical techniques have been in all cases not-destructives (X-ray diffraction and fluorescence spectroscopy, infrared spectroscopy, optical microscopy and environmental electronic microscopy, with microanalysis). The archaeometric analysis results show a mineralogical nature of these materials, based in variscite and amber. The textural and compositional features are very homogeneous between each assemblage of beads. The importance of the alteration phenomena and dissolution of the archaeological objects are determined and quantified. This alteration is due to an intense chemical attack during the burial periods. The possible source areas of provenance for these materials are also discussed on the basis of geochemical data obtained in the analysis.

Palabras clave: Arqueometría; Variscita; Ámbar; Pulimentos; Neolítico; Megalitismo; Galicia; DRX; EFRX; MEBA; EEDRX; EITF.

Key words: Archaeometry; Variscite; Amber; Polished industry; Neolithic; Megalithism; Galicia; XRD; WDXRF; ESEM; EDS; FTIR.

\section{LA EXCAVACIÓN DEL TÚMULO}

Los trabajos arqueológicos en el túmulo 1 de Chousa Nova, Silleda, norte de la provincia de Pontevedra (Fig. 1), estuvieron motivados por las obras de construcción de la Línea de Alta Velocidad Ourense-Santiago, tramo Lalín-Santiago y 


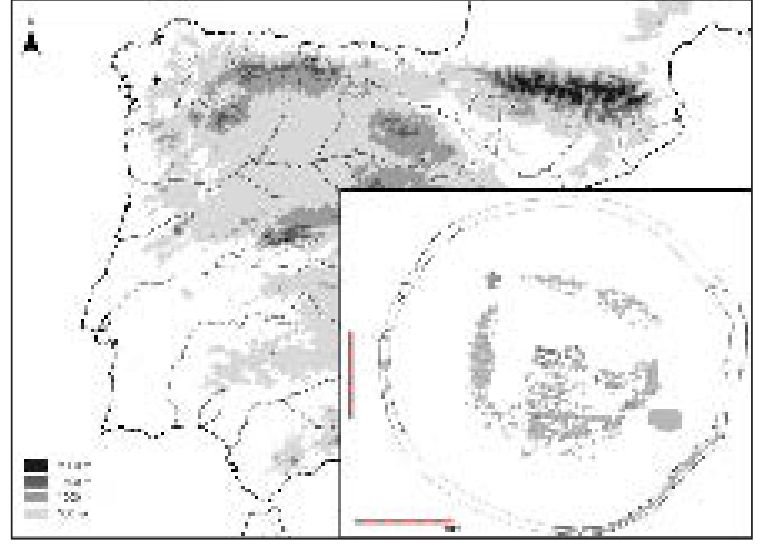

Fig. 1. Mapa de situación del yacimiento de Chousa Nova (Silleda, Pontevedra) y plano sintético de la excavación.

subtramo Carboeiro-Dornelas, Silleda(1). Las obras previstas en la zona provocaban la total destrucción del yacimiento, así como la completa alteración de su entorno inmediato. La dificultad de modificar puntualmente el trazado de la línea ferroviaria, para alejarla del túmulo, unida al estado de deterioro del yacimiento, determinó su excavación integral, así como una intervención arqueológica sistemática, con sondeos en su entorno.

Una pista asfaltada había arrasado, aparentemente, todo el sector norte del túmulo, que mostraba la masa tumular muy rebajada debido a los trabajos agrícolas y forestales realizados durante años en la zona. Una gran depresión, visible en lo que parecía el sector central del monumento, evidenciaba que había sido expoliado con anterioridad a la construcción de la pista asfaltada. La agresión más reciente era un poste de tendido eléctrico colocado sobre el túmulo.

El avance de los trabajos de excavación permitió descubrir un yacimiento que poco tenía que ver con su aspecto superficial. La pista asfaltada había seccionado únicamente un cuarto del túmulo, la masa tumular se conservaba en un relativo buen estado y la arquitectura del monumento era de una gran complejidad, con anillo perimetral, evidencias de dos momentos de tumulación, cámara y estructura de acceso (Fig. 2).

(1) La empresa encargada de este tramo de la L.A.V. Ourense-Santiago fue la UTE Docarbo. El Administrador de Infraestructuras Ferroviarias (ADIF), promotor de las obras, financió la totalidad de los trabajos arqueológicos.

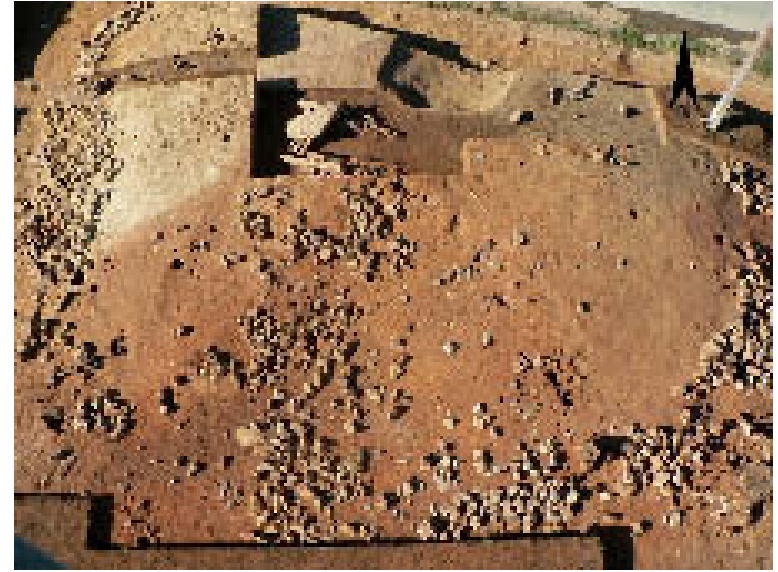

Fig. 2. Vista aérea del túmulo de Chousa Nova durante el proceso de excavación.

Bajo el yacimiento, y rodeándolo, aparecieron una serie de estructuras excavadas en el sustrato que indicaban una clara actividad antrópica previa a la construcción del túmulo. Elementos de este tipo están localizándose en los últimos años en Galicia, a medida que aumenta la superficie de intervención en el entorno de los túmulos (Vilaseco 2006). Una zanja perimetral rodeaba al yacimiento, creando un círculo concéntrico a éste, situado a una distancia constante de unos $8 \mathrm{~m}$ de su base.

La zanja fue identificada durante los trabajos de control y seguimiento, una vez finalizada la excavación en área. Presentaba una clara apertura en el sector este-sureste, mostrando en su interior, especialmente en la zona sur, una acumulación lineal de pequeñas piedras que formaban un anillo externo concéntrico al del túmulo. La zanja estaba muy alterada y únicamente era identificable en el contacto con el sustrato. Su impronta se perdía en los sectores más arrasados por trabajos agrícolas, pero, en cambio, se definía claramente bajo la pista asfaltada.

Dentro del espacio marcado por la zanja, y en algunos casos bajo la propia masa tumular, se localizaron otras zanjas y fosas de difícil interpretación. En el interior de una de ellas que recorría longitudinalmente el sector sur del yacimiento apareció una bola de ocre. Estaba cubierta por un material que era idéntico al substrato en el que estaba excavada. Al sureste y este del túmulo se situaban dos zanjas tangenciales a él, de sección en U muy abierta. En su base se dis- 
ponía una hilera de piedras de tamaño medio y en su interior se localizaron algunos líticos tallados. Al noroeste del túmulo apareció una fosa de planta circular, rellena por piedras de pequeño tamaño que creaban una superficie plana dispuesta sobre una tierra con evidentes restos de combustión. Parece una estructura tipo parrilla, similar a las localizadas en poblados prehistóricos gallegos como los de Monte dos Remedios (Fábregas et al. 2007), Gándara, Cartas de Vilar o Porto dos Valos, con una amplitud cronológica que abarca desde los inicios del Neolítico en la región hasta momentos avanzados del Calcolítico (Lima 2000).

Un anillo pétreo ceñía la base del túmulo y en algunos sectores se conservaban restos de una coraza que parece corresponderse con una primera fase de tumulación. Los dos momentos de tumulación eran perfectamente visibles en la secuencia estratigráfica, especialmente en el sector oeste del túmulo. La masa tumular estaba compuesta por tierras procedentes del entorno inmediato al yacimiento, con una increíble variedad de colores (rojos, amarillos o grises), que parecían prolongar, sobre la superficie del túmulo, la variedad existente en el propio sustrato, ya que su situación se correspondía fielmente con su disposición sobre el terreno. Bajo ellas, y directamente sobre el sustrato, se extendía un fino nivel de una tierra de gran dureza que interpretamos como un horizonte de trabajo creado durante las labores de acondicionamiento de la zona en el momento de construcción del monumento.

En el interior del túmulo se conservaba una cámara megalítica, aparentemente intacta, que presentaba, además, una gran piedra al noreste de la entrada. Esta piedra parece formar parte de una estructura de acceso que se extiende al este-sureste de la cámara. Este posible acceso estaba constituido por dos líneas de piedras de mediano tamaño que finalizaban, frente a la entrada de la cámara, en una acumulación de piedras apoyada contra la gran piedra citada. No parece presentar similitud con ninguna de las estructuras de acceso conocidas en el noroeste de la Península Ibérica (Vilaseco 1997/98). La disposición de las piedras no parece garantizar un paso adecuado desde el exterior del túmulo hasta la cámara.

La cámara estaba integrada por cinco ortostatos que creaban un espacio abierto hacia el estesureste. Su escasa altura otorgaba al monumento un cierto aspecto cistoide. Los ortostatos estaban rodeados de una tierra arcillosa compactada, un contrafuerte térreo que fortalecía la estructura pétrea. Este tipo de estructuras de refuerzo resultan habituales en el megalitismo noroccidental, aunque suelen realizarse en piedra. La gran losa de cubierta apareció fracturada (Fig. 3). Dos grandes pedazos ocupaban el interior de la cámara mientras que uno de menor tamaño permaneció in situ. Estos restos estaban perfectamente tapados por tierra tumular perteneciente a la segunda fase de tumulación. Su dureza contrastaba con la tierra suelta que llenaba el espacio de la cámara situado bajo el fragmento de cubierta conservado in situ. Una vez retirada la cubierta del interior de la cámara pudo comprobarse que la piedra de cabecera y el quinto ortostato estaban realizados en un material de muy mala calidad, blando y metamorfizado, con gran cantidad de micas. Estas lajas posiblemente fuesen elegidas, en lugar del granito de mejor calidad del resto de ortostatos, por el intenso brillo que presentan. El deterioro de dichas piedras complicaba la estabilidad de la cámara y parece haber sido la causa de la rotura de la cubierta cuando el monumento aún estaba en uso.

El interior de la cámara estaba aparentemente intacto: un fino nivel de tierra de aspecto orgánico y pequeñas piedras planas lo cubrían. Un nivel de piedras similar se ha constatado en otras intervenciones, como en la pequeña cámara, aparentemente intacta, del túmulo de Forno dos Mouros 5 (Mañana 2005), o en el dolmen de

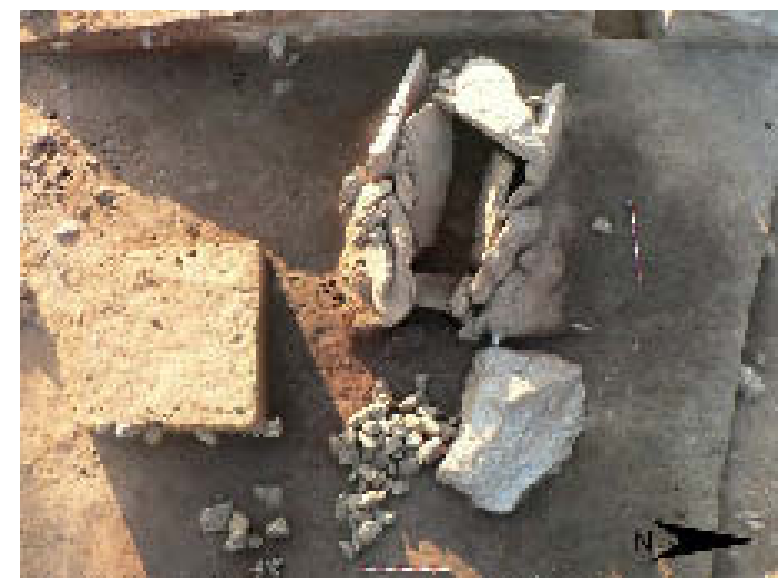

Fig. 3. Detalle de la cámara del túmulo 1 de Chousa Nova con parte de la piedra de cubierta fracturada en su interior. 
corredor de Dombate, donde, pese a estar expoliado, fue posible distinguir este nivel en la zona intacta próxima a los ortostatos (Alonso y Bello 1995).

En el sector central de la cámara, bajo varias pequeñas piedras planas, se localizaron una serie de cuentas de diferentes tamaños, material y tipología, dispuestas secuencialmente para formar un collar. Las de mayor tamaño se situaban en el centro y hacia ambos lados, en dirección a la losa de cabecera, otras cuentas cilíndricas cuyo tamaño disminuía progresivamente hasta disponerse claramente como un collar colocado sobre un pecho humano (Fig. 4).

El aspecto orgánico de algunas de las cuentas de mayor tamaño, su mal estado de conservación y su evidente similitud con elementos de ámbar aconsejó su extracción, junto con la tierra que las rodeaba, para facilitar su conservación y consolidación. Los posteriores análisis confirmaron que se trataba de este material, desconocido hasta el momento en contextos tan antiguos del noroeste peninsular.

La disposición de las cuentas y del resto de ajuar procedente del interior de la cámara permite pensar en un enterramiento individual, aunque no fue posible recuperar ningún resto óseo ni dental, pese a recogerse y procesarse la totalidad de la tierra del interior de la cámara. La falta de restos orgánicos resulta algo habitual en este sector del noroeste de la Península Ibérica

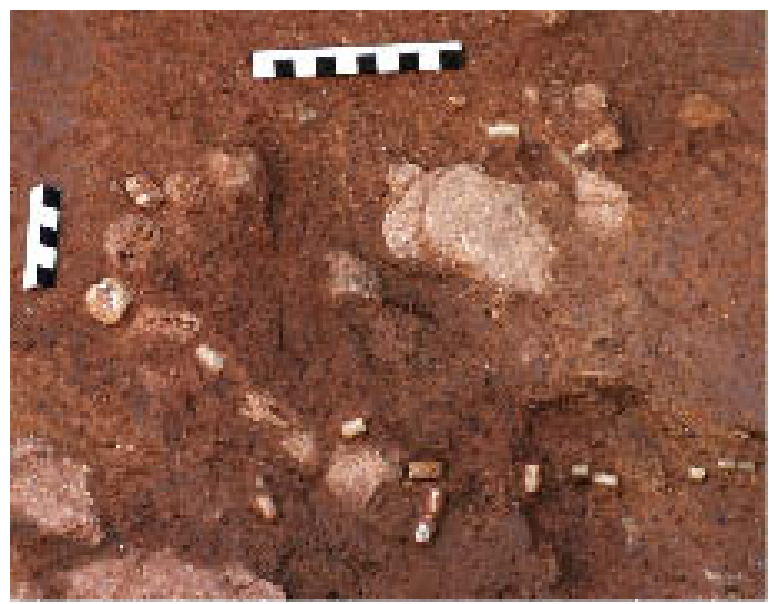

Fig. 4. Disposición de las cuentas del collar, durante el proceso de excavación de la cámara del túmulo 1 de Chousa Nova.

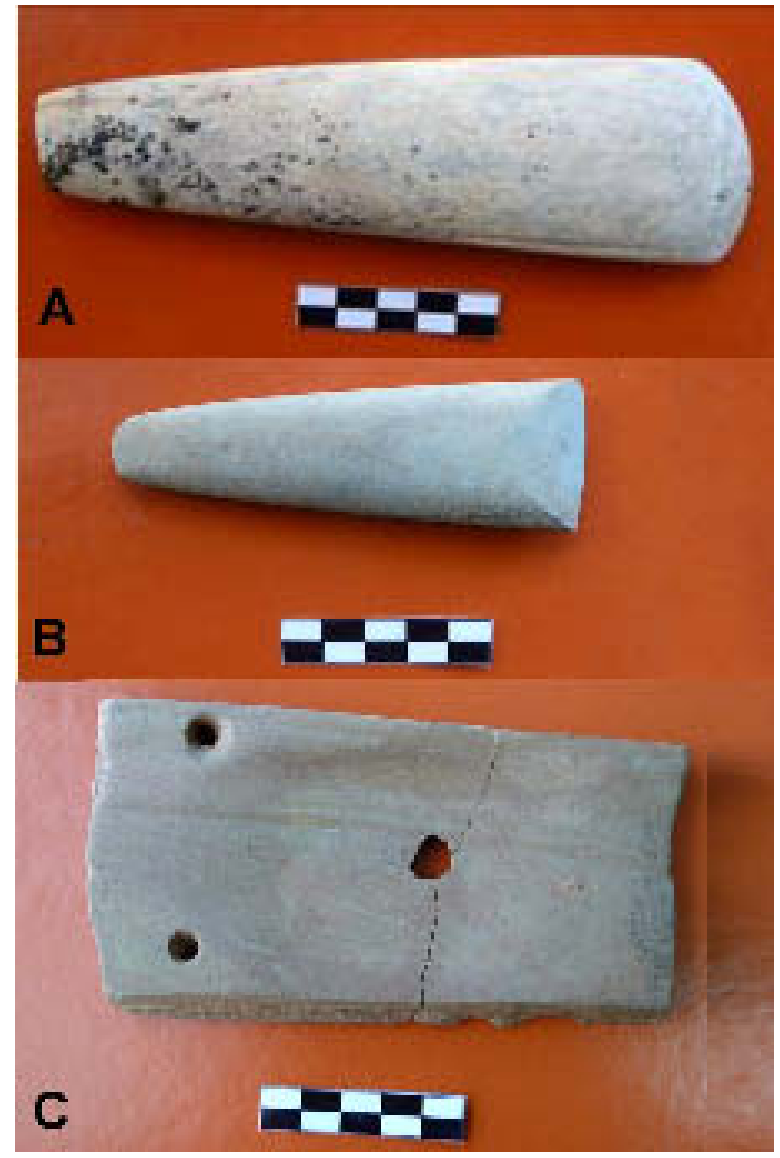

Fig. 5. Túmulo 1 de Chousa Nova. A: Lámina de azada en sillimanita; B: hacha en roca metamórfica y C: afilador en arenisca de grano muy fino, recuperados en la cámara del dolmen.

y está causado por la altísima acidez del terreno. Teniendo en cuenta la disposición del ajuar podemos suponer que el cadáver fue colocado en decúbito supino, con el collar sobre el pecho; una lámina de azada se situaba a escasa distancia de su cabeza, en paralelo a la piedra de cabecera (Fig. 5A); un pequeño cincel próximo a su mano derecha (Fig. 5B) y por último, un gastado afilador de piedra de forma rectangular, situado a sus pies, junto a la entrada de la cámara (Fig. 5C).

La posible disposición del cuerpo (Fig. 6), la presencia de un elemento pulido que por sus características y aparente falta de uso puede considerarse como de prestigio, junto a las cuentas de variscita, evocan vagamente los enterramientos del túmulo de Illade 0 (Vaquero 1999), monu- 


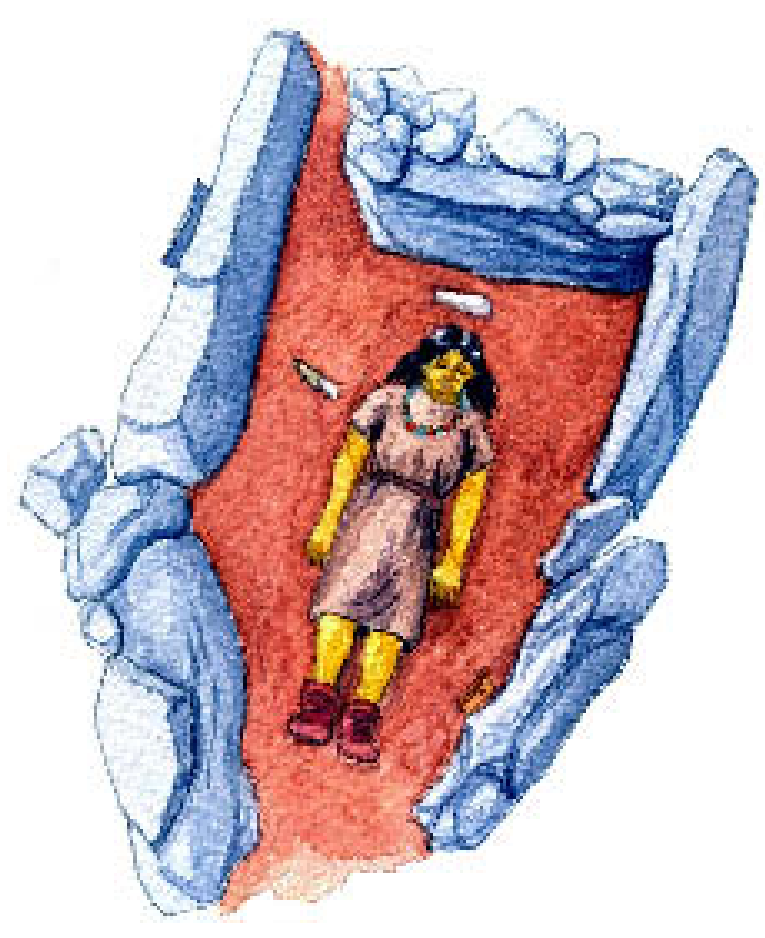

Fig. 6. Reconstrucción hipotética del enterramiento del túmulo 1 de Chousa Nova.

mento que se remonta a un momento inicial del megalitismo gallego.

Los resultados de carbono 14 obtenidos hasta el momento para el túmulo 1 de Chousa Nova parecen refrendar esta similitud. Uno de los tres carbones de gran tamaño localizados sobre el horizonte de trabajo existente bajo la tumulación se dató en $5450 \pm 40$ BP (Beta-277240), 43504240 Cal BC (calibración del laboratorio a partir de la curva de calibración INTCAL04, Reimer et al. 2004). La situación, forma y dimensiones de estos pequeños troncos carbonizados impiden considerarlos un simple aporte casual. Por el momento sólo se cuenta con otra datación procedente de un depósito de tierra con pequeños carbones localizado bajo la fosa de cimentación de la cámara, directamente sobre el sustrato. Su resultado, $9830 \pm 50$ BP (Beta-277239), 9350-9240 Cal BC, dada su gran antigüedad, no puede ponerse en relación con la estructura tumular. Probablemente se trate de restos de una antigua raíz o algún tipo de intrusismo aportado, posiblemente de forma accidental, durante la construcción de la cámara.

\section{METODOLOGÍA}

La espectacularidad de los materiales recuperados, en comparación con la sobriedad habitual del registro megalítico galaico, unida a la excepcionalidad del ámbar en este contexto, aconsejó el análisis individual de cada elemento del ajuar.

Hemos utilizado métodos analíticos habituales en el estudio de industrias líticas, selectivos para cada tipo de cuenta. En las variscitas se utilizó Difracción de rayos X y siempre de forma directa sobre el objeto, para que no fueran destructivos. Igualmente se han realizado análisis químicos elementales cualitativos y cuantitativos directamente sobre las cuentas de variscita, mediante Espectrometría por Fluorescencia de rayos $\mathrm{X} W D X R F$, utilizando el equipo Pioneer S-4 de los Servicios Centrales de Ciencia y Tecnología (SCCYT) de la Universidad de Cádiz. Además, se usó la Microscopía Electrónica de barrido ambiental ESEM, concretamente un equipo Philips Quanta 200, de la UCA, operando en condiciones de Low vacuum, para las cuentas de variscita.

Microscopía óptica: todas las muestras han sido examinadas mediante estereomicroscopía óptica para el estudio de superficies, fenómenos de alteración, presencia de inclusiones minerales, alteraciones antrópicas de las cuentas (reciclado de cuentas de collar), desgastes asimétricos, etc. Todas estas observaciones han sido registradas digitalmente en una base de datos fotográfica de dichos materiales arqueológicos.

Difracción de rayos X: en especial el método de polvo policristalino, es ideal para la identificación de fases minerales o de compuestos orgánicos o inorgánicos. Las muestras, que normalmente han de ser preparadas en polvo para esta técnica, en este caso y para hacer el análisis nodestructivo, fueron introducidas directamente en un difractómetro Bruker D-8 Advance, de la UCA, con ciertas modificaciones en la configuración de los portamuestras. Los datos se han adquirido y procesado con el programa DiffractPlus y EVA de BRUKER-AXS, identificando las fases minerales mediante las fichas de la base de datos ASTM (American Society for Testing and Materials).

Se ha utilizado para los análisis radiación $\mathrm{Cu}$ $\mathrm{K} \alpha$ filtrada con $\mathrm{Ni}$, monocromador de grafito $\mathrm{y}$ rendijas fijas, con ángulos de barrido 2 theta entre $5^{\circ}$ y $50^{\circ}$. 
Espectroscopía por Fluorescencia de rayos X (WDRXF): esta técnica de rayos $\mathrm{X}$ constituye un buen método para los análisis cuantitativos de elementos mayoritarios y minoritarios en muestras sólidas. Igualmente se pueden estudiar, siempre que su tamaño lo permita $(>40 \mathrm{~mm})$, en el equipo de la División de Rayos X de la UCA. Se utilizó un Espectrómetro por Dispersión de Onda Secuencial (WDFRX), modelo S-4 Pioneer, de Bruker-AXS, con un tubo de $\mathrm{Rh}$, operando a $4000 \mathrm{~W}$ y usando, bien el material pulverizado y prensado en un disco plano o bien un pellet, fundido en una "perla" de muestra en polvo, mezclada con el $50 \%$ de $\mathrm{Li}_{2} \mathrm{~B}_{4} \mathrm{O}_{7}$ y el $50 \%$ de $\mathrm{LiBO}_{2}$. Las "perlas" normalmente producen unos resultados analíticos más precisos que los pellets prensados o la muestra expuesta directamente, ya que esta técnica supera algunos de los problemas asociados con los efectos de superficie, de mineralogía o de variación del tamaño de partícula. Dado que los análisis de estos materiales arqueológicos debían ser no-destructivos, en la mayoría de los casos hemos usado directamente la muestra arqueológica. La única condición es que presentara una superficie plana.

Microscopía electrónica de barrido ambiental (ESEM): en esta variante de la técnica de análisis empleamos condiciones de trabajo de bajo vacío, lo que nos permite el uso de muestras sin metalizar. Este factor ha sido importante, para preservar totalmente los materiales arqueológicos sin alteración.

Las muestras se montaron en portamuestras de aluminio, con cinta conductora doble adhesiva. El equipo utilizado fue el citado microscopio Philips Quanta 200, trabajando a $22 \mathrm{KV}$ y en modo Low Vacuum.

Espectroscopía por Energía Dispersiva de Rayos X $(E D S)$ : nos ha permitido obtener análisis puntuales de las muestras, simultáneamente a su observación mediante ESEM. Se utilizó un equipo EDAX Genesis Spectrum, conectado al microscopio electrónico Quanta 200.

Espectroscopía de Infrarrojos por Transformada de Fourier (FTIR): esta técnica requiere tan sólo 2-3 mg de muestra y ha sido utilizada en el análisis de las cuentas de material orgánico que suponíamos de ámbar, tras un primer análisis visual y con estereomicroscopía. La metodología ha sido la típica: elaboración de pastillas de 200 $\mathrm{mg}$ de $\mathrm{KBr}$, finamente molturadas en mortero de ágata, con $4 \mathrm{mg}$ de muestra y perfectamente mez- cladas, para posteriormente ser prensadas a 12 $\mathrm{Tm} / \mathrm{cm}^{2}$, durante unos minutos. Se ha utilizado un equipo de espectroscopía de infrarrojos por Transformada de Fourier, marca Bruker Alpha, en un modo IR transmitancia, para un intervalo de 450 y $4.000 \mathrm{~cm}^{-1}$. Se realizaron cinco barridos por muestra, cuyos resultados fueron promediados, mediante el programa OPUS. Los espectros de las 3 muestras de ámbar analizadas aparecen en la figura 7. No descartamos futuros análisis mediante Espectroscopía Raman, Espectroscopía Infrarroja Directa (Teodor et al. 2010) o técnicas de Cromatografía con Pirolisis PY-GC-MS (Anderson et al. 1992), que permitan ampliar las informaciones sobre éstos u otros ámbares de la Península Ibérica que estudiamos en la actualidad.

Estos materiales que, como se ha comentado, se presentaron completamente alterados en la excavación, fueron consolidados y extraídos con el máximo cuidado, dado su delicado estado de con-

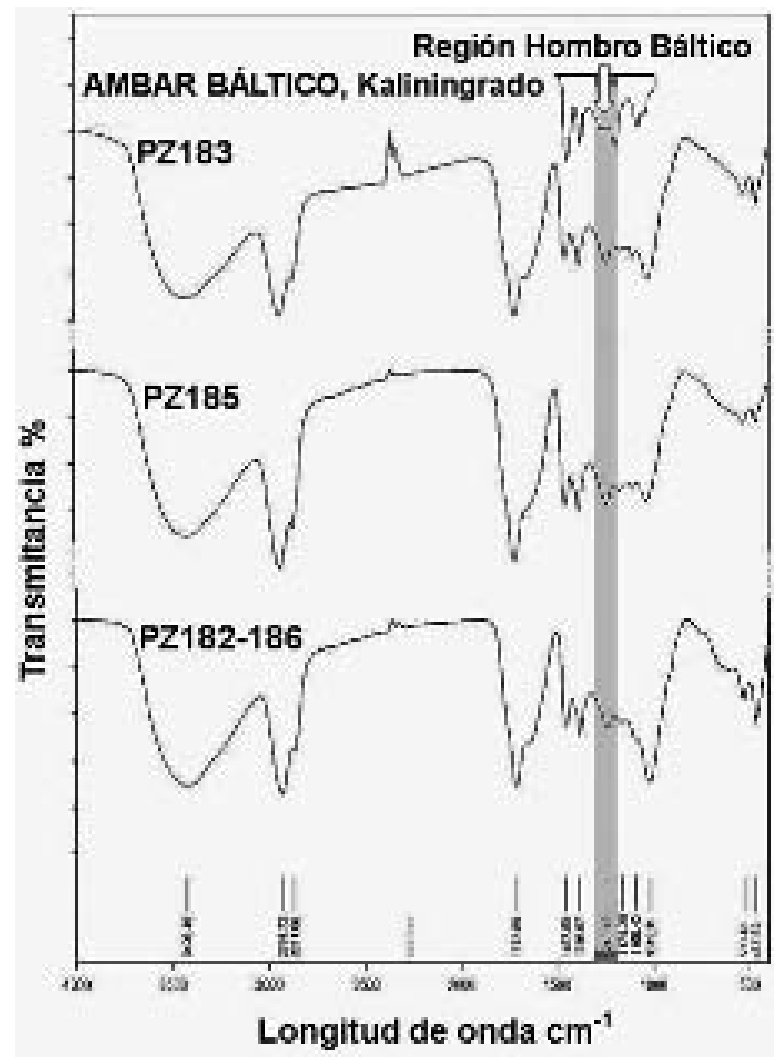

Fig. 7. Espectros FTIR de tres de las cuentas de ámbar de Chousa Nova y detalle del "hombro báltico" en una muestra de ámbar báltico de Kaliningrado. 
servación. Para estos análisis se utilizaron algunos de los pequeños fragmentos desprendidos de las cuentas y recuperados en su totalidad durante este delicado proceso de excavación, previa a su consolidación in situ.

\section{RESULTADOS}

La excavación del sector central del túmulo de Chousa Nova 1 localizó una pequeña cámara megalítica integrada por cinco ortostatos que creaban un espacio abierto hacia el este-sureste. En su interior se encontraron los restos de una gran losa de cubierta completamente cubiertos por tierra de la segunda fase de tumulación del yacimiento. Ello permite suponer un tapado intencional de la cámara con la cubierta ya fracturada. Si la datación 4350-4240 Cal BC se confirma en posteriores análisis $\mathrm{C} 14$ estaríamos ante una de las cámaras megalíticas más antiguas, de las localizadas hasta el momento, en el noroeste peninsular (Fábregas y Vilaseco 2006).

El interior de la cámara estaba aparentemente intacto, aislado por la tierra de la segunda tumulación y los fragmentos de la losa de cubierta. El nivel de uso de la cámara estaba formado por un fino depósito de tierra de aspecto orgánico y un nivel de pequeñas piedras planas. En este nivel se localizó el ajuar, formado por un afilador, un pequeño cincel y una lámina de azada que, tras su análisis mediante DRX directa, ha resultado ser de sillimanita (variedad fibrolita).

Ocupando el sector central de la cámara y bajo el nivel de pequeñas piedras planas, apareció un collar formado por cuentas de variscita y ámbar con diferentes tamaños y tipología. La distribución de las cuentas aparentaba un collar colocado en su posible posición original, sobre un pecho humano (Fig. 4). Sólo se conoce en la bibliografía europea un collar completo, con presencia simultánea de ambos materiales, en el dolmen de Alberite I, Villamartín, Cádiz (Domínguez-Bella y Morata 1995).

Las 6 cuentas de material ambarino, completamente deterioradas, fueron cuidadosamente recuperadas durante el proceso de excavación. Sus tamaños son ligeramente superiores a las de variscita, oscilando entre los $3,2 \mathrm{~cm}$ y $\operatorname{los} 1,7 \mathrm{~cm}$ de longitud. Dentro del conjunto se alternaban con cuentas de color verde de la zona baja y central del collar.
Durante la excavación fue muy difícil la identificación de la naturaleza de este material orgánico de aspecto terroso, si bien se pudo reconstruir la posición de cada cuenta, su forma y su tamaño. El aspecto orgánico, su mal estado de conservación y su evidente similitud con elementos de ámbar aconsejó su extracción completa, junto con la tierra que las rodeaba, para facilitar su posterior consolidación y estudio arqueométrico. Este material es desconocido hasta este momento en contextos tan antiguos del noroeste peninsular.

Se prepararon muestras de dichas cuentas y se analizaron mediante FTIR, resultando ser todas de ámbar e idénticas. Pero falta el "hombro báltico", una inflexión característica, que define una zona más o menos plana y casi horizontal, en la región de 1.200-1.300 $\mathrm{cm}^{-1}$ dentro del espectro de infrarrojos (Savkevich y Shaks 1964) (Fig. 7 arriba) y que aparece en exclusiva en los ámbares tipo succinita de los yacimientos de edad terciaria de la región del mar Báltico (Beck et al. 1965). Como las cuentas no tienen una composición del tipo succinita, el ámbar no es de origen báltico. Sus espectros de infrarrojos (Fig. 7) presentan bandas a: 1.714, 1.457, 1.386, 1.243, 1.174, $1.106,1.019,533$ y $467 \mathrm{~cm}^{-1}$, que deben corresponder a vibraciones típicas de grupos $\mathrm{C}=\mathrm{C}$, $\mathrm{C}=\mathrm{O}, \mathrm{C}=\mathrm{N}, \mathrm{C}-\mathrm{C}, \mathrm{C}-\mathrm{O}$ y $\mathrm{C}-\mathrm{N}$, además del grupo $\delta \mathrm{H}_{2} \mathrm{O}\left(1.635 \mathrm{~cm}^{-1}\right)$.

Estos resultados son muy similares a los espectros de ámbares arqueológicos obtenidos en las cuentas de collar del citado dolmen de Alberite I, Villamartín, Cádiz (Ramos y Giles 1996; Domínguez-Bella y Morata 1995; DomínguezBella et al. 1998) identificados como simetita (Domínguez-Bella et al. 2001), así como a los aparecidos en yacimientos prehistóricos del megalitismo de Portugal (Vilaça et al. 2002: Fig. 7). Se están comparando con otros ámbares arqueológicos de reciente aparición como los que estamos estudiando en la Bahía (Vijande 2009) y la Sierra de Cádiz. En cambio, resultan algo diferentes o, al menos, no idénticos, a los ámbares arqueológicos y geológicos citados en la cornisa cantábrica (Álvarez Fernández et al. 2005).

El collar está compuesto, además, por 29 cuentas cilíndricas o barriliformes de color verde y verde-azulado, que tras su análisis directo mediante Difracción de rayos $\mathrm{X}$ han resultado ser todas de variscita tipo Messbach/Zamora (fichas 00-025-0018 y 00-025-0019 JCPDS) con presencia de metavariscita y cuarzo (Fig. 8) (Arribas et 


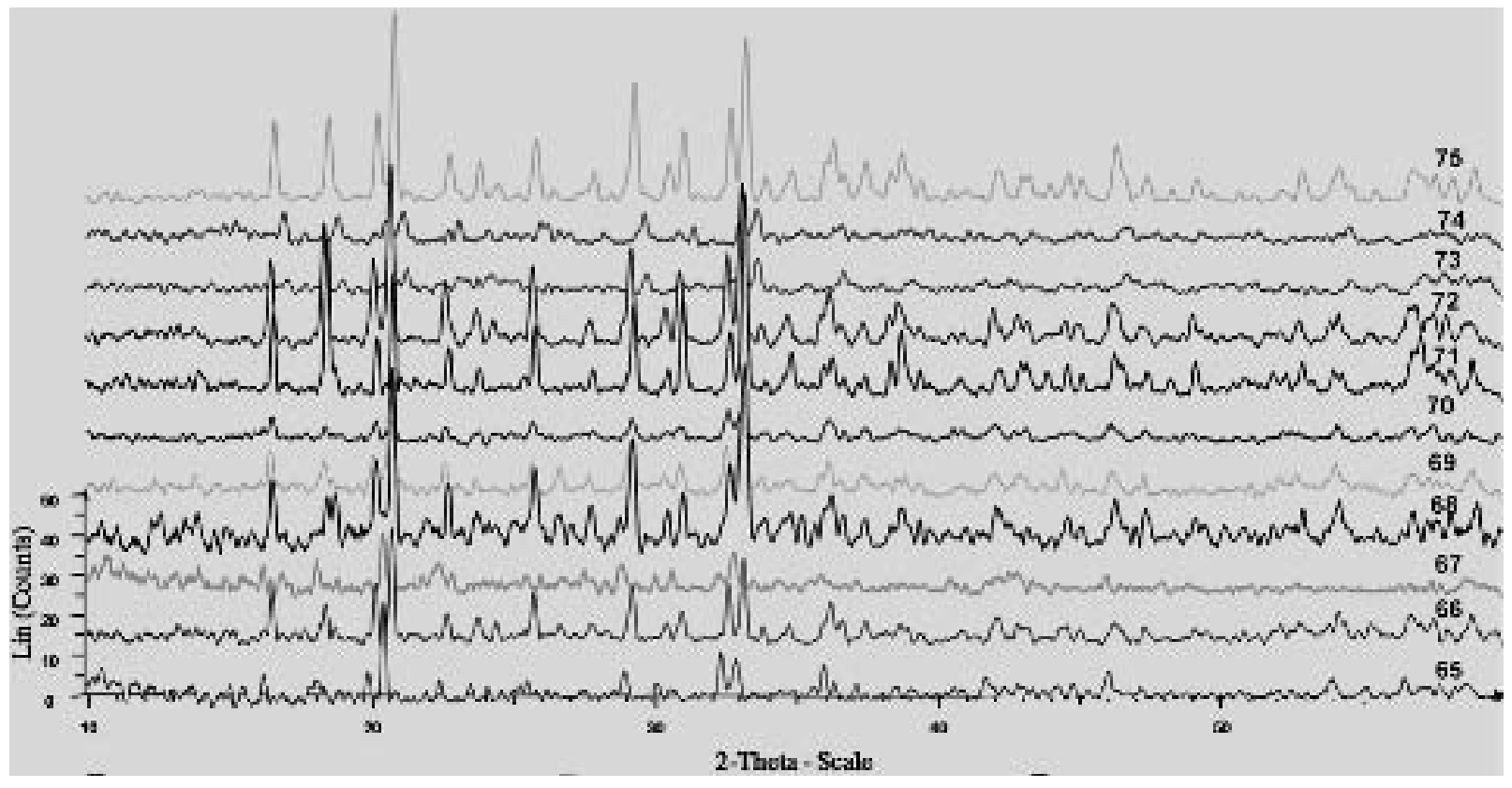

Fig. 8. Difractogramas de Rayos X de las cuentas de collar de variscita del túmulo 1 de Chousa Nova.

al. 1971). Los análisis no-destructivos mediante WDFRX directa reflejan una homogeneidad de composición (Tab. 1) que nos indica un origen geológico común. La estamos comparando actualmente con la de las muestras geológicas que hemos recogido los últimos 15 años en el suroes-

\begin{tabular}{|c|c|}
\hline Elementos & \% \\
\hline $\mathrm{P}_{2} \mathrm{O}_{5}$ & 28,87 \\
$\mathrm{Al}_{2} \mathrm{O}_{3}$ & 17,22 \\
$\mathrm{SiO}_{2}$ & 1,58 \\
$\mathrm{Fe}_{2} \mathrm{O}_{3}$ & 3,33 \\
$\mathrm{MgO}$ & 0,17 \\
$\mathrm{Na}_{2} \mathrm{O}$ & 0,13 \\
$\mathrm{~K}_{2} \mathrm{O}$ & 0,04 \\
$\mathrm{SO}_{3}$ & 0,01 \\
$\mathrm{TiO}_{2}$ & 0,123 \\
$\mathrm{~V}_{2} \mathrm{O}_{5}$ & 0,10 \\
$\mathrm{Cr}_{2} \mathrm{O}_{3}$ & 0,101 \\
$\mathrm{CaO}$ & 0,1285 \\
$\mathrm{Cl}$ & 0,0106 \\
$\mathrm{MnO}$ & 0,0016 \\
$\mathrm{SrO}$ & 0,0033 \\
\hline
\end{tabular}

Tab. 1. Análisis químico cuantitativo promedio de las cuentas de variscita de Chousa Nova, a partir de WDFRX. te europeo y, en especial, en la Península Ibérica (Domínguez-Bella 2004)(2). Los primeros resultados en elementos como $\mathrm{Cr}$ y V sugieren un origen en yacimientos del Oeste peninsular como Encinasola (Huelva) (Domínguez-Bella et al. 1998; Domínguez-Bella et al., 2003) (3) o la comarca de Aliste (Zamora) (Domínguez-Bella 2004), aún por precisar, en base a los análisis que ya están en fase de finalización y publicación.

La superficie de estas cuentas presenta una fuerte alteración, muy visible mediante su análisis con Microscopía óptica (Fig. 9A) y Microscopía electrónica de barrido (Fig. 9B). En algunas cuentas se conservaron restos de la superficie original, lo que nos permitió cuantificar la alteración a una profundidad en la muestra de unas $250 \mu \mathrm{m}$ (Fig. 9B). Se observan además fisuras e inclusiones de roca y minerales en algunas cuentas. El color original es muy visible en las zonas de rotura de

(2) Domínguez-Bella, S.; Morata, D.; Rosa, J. de la y Ramos Muñoz, J. 2002: "Neolithic Trade Routes in SW Iberian Peninsula? Variscite green beads from some Neolithic sites in the Cádiz Province (SW Spain): Raw materials and provenance areas". Proceedings of 32 International Symposium on Archaeometry 2000. México D.F. 10 pp. Electronic Book. Instituto Investigaciones Antropológicas. Universidad Nacional Autónoma de México (URL en trámite).

(3) Véase n. 2. 


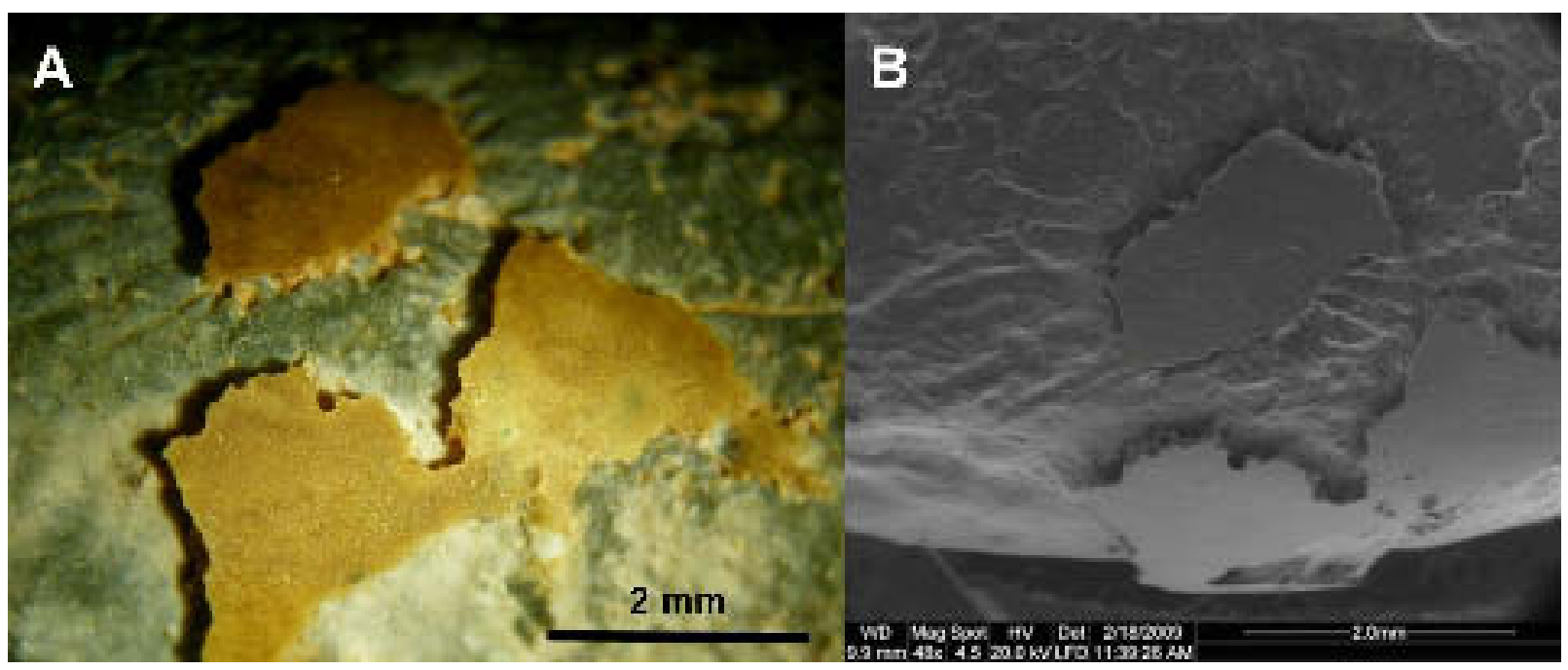

Fig. 9. Túmulo 1 de Chousa Nova: A. Vista de la superficie de una de las cuentas de variscita, representativa del alto grado de alteración superficial que presentan; B. Vista mediante ESEM de la superficie de la cuenta de variscita anterior.

una de ellas. Aún más interesante es el proceso de reciclaje en otra, tras su rotura en época prehistórica, ejecutando un anillo ecuatorial por rebaje (detalle en Fig. 10A) para poder ser atada de nuevo en el collar. La perforación de las cuentas fue por doble taladrado con broca prismática (Fig. 10B). En las de mayor tamaño se ha observado un desgaste diferencial que pudo estar producido por el cordón del collar. Ello resultaría de un uso muy prolongado del mismo o de un fallo en el proceso de perforación (Fig. 10C).

La disposición original del collar ha sido muy bien definida tras el proceso de excavación y registro con coordenadas UTM de cada una de las cuentas. Las de variscita y ámbar se alternan, tal y como se observa en nuestra propuesta de reconstrucción (Fig. 11).

\section{CONCLUSIONES}

A modo de resumen, es importante subrayar que estamos ante un trabajo en curso y a la espera de la realización de nuevas analíticas. Los resultados principales son:

- Se analizan por primera vez muestras de ámbar arqueológico en un monumento funerario del megalitismo de Galicia con una datación absoluta ya determinada en $5450 \pm 40 \mathrm{BP}, 4350$ 4240 Cal BC (Beta-277240).
- Se caracteriza arqueométricamente el mayor conjunto conocido de cuentas verdes de Galicia, resultando ser en su totalidad variscitas.

-En base a la bibliografía que conocemos, se trataría de la segunda cita europea de un collar recuperado in situ con una cronología del V milenio A.C., que combina cuentas de variscita y ámbar, componentes exclusivos del collar, a diferencia del de Alberite I, Villamartín, Cádiz.

- Se realizan análisis directos no-destructivos mediante $F R X$ de muestras arqueológicas de variscita.

- Se establece el posible origen de las variscitas en yacimientos geológicos del occidente de la Península Ibérica.

- Se determinan aspectos tecnológicos en las cuentas de variscita (dobles perforaciones, huellas de uso) inéditos hasta la fecha, que confirman la reutilización de este material como joya $\mathrm{u}$ objeto de prestigio, aun en el caso de que se fracturara durante su uso.

- Se constata un intenso proceso de alteración de los restos arqueológicos orgánicos e inorgánicos (desaparición total de restos óseos y fuerte alteración de ámbar y variscitas).

- Las cuentas de ámbar no proceden del Báltico. Su origen posiblemente está en la Península Ibérica. En general, están muy alteradas y son de colores rojizos. Se han comparado estas muestras con ámbares geológicos de toda la Península Ibé- 


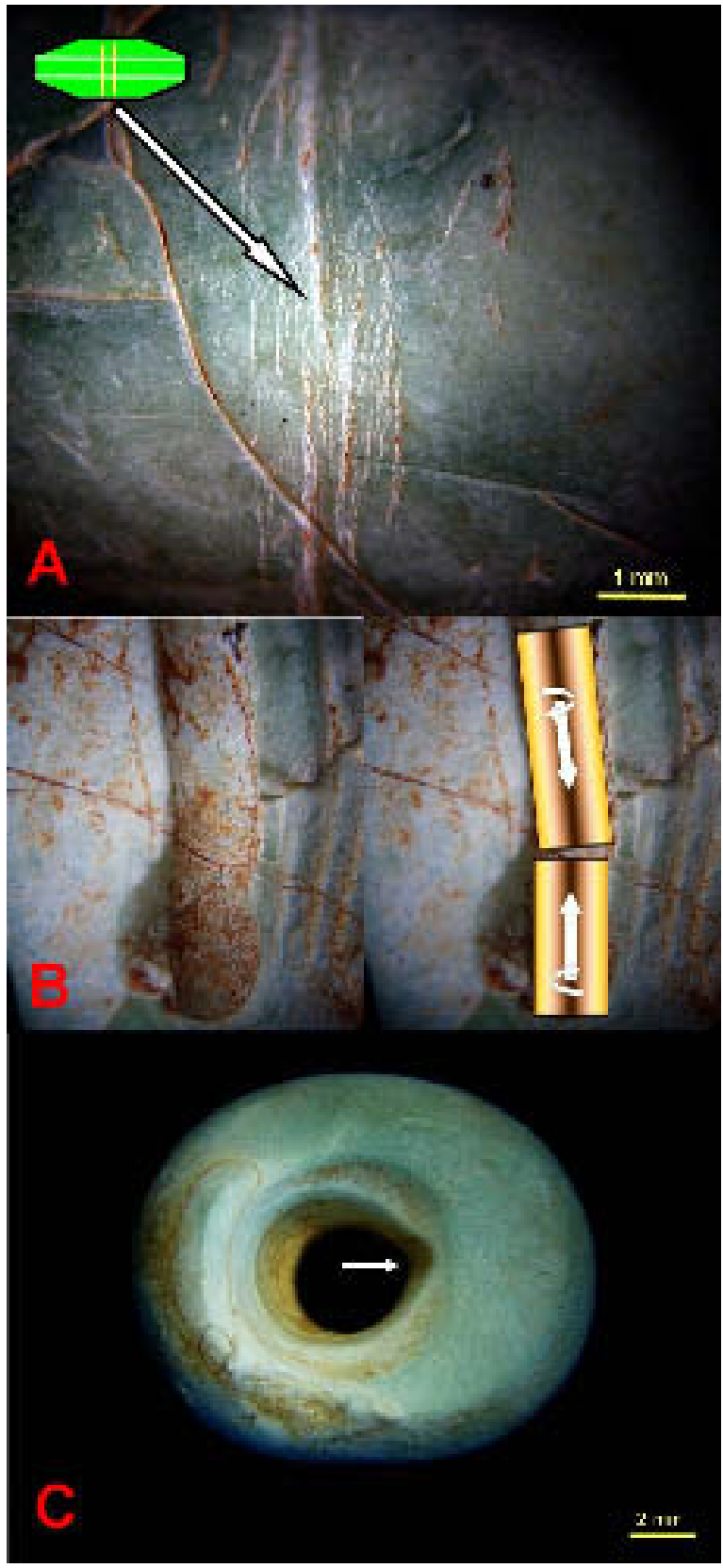

Fig. 10. Túmulo 1 de Chousa Nova. A. Cuenta de variscita fragmentada reutilizada ejecutando un anillo ecuatorial para su enlazado; B. Reconstrucción del proceso de taladrado de una cuenta, mediante una doble perforación; C. Cuenta de variscita con desgaste desigual en el interior de su perforación central.

rica, además de los referentes del Báltico. No se tiene constancia hasta la fecha de ningún yacimiento geológico de ámbar en Galicia, lo que concuerda bastante bien con sus características

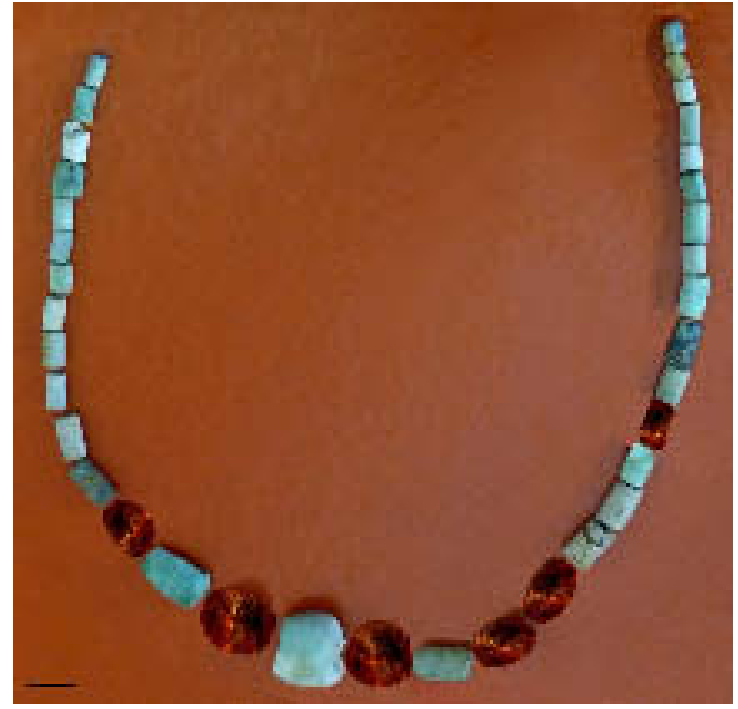

Fig. 11. Reconstrucción propuesta para el collar de variscita y ámbar de Chousa Nova (barra de escala $=1 \mathrm{~cm}$ ).

geológicas. Su posible origen debe ser, al menos, externo a este territorio.

- Se localiza en el noroeste peninsular el primer enterramiento megalítico sellado e intacto, dentro de una cámara no expoliada.

- En el interior del monumento había un enterramiento (recreado en la Fig. 6). El individuo llevaba al cuello un collar. A partir de la información arqueológica y arqueométrica obtenida, se propone una reconstrucción de su aspecto original (Fig. 11), basada en el registro tridimensional realizado durante la intervención, la posición de cada una de las cuentas, su tamaño, su estado de conservación en el momento de la excavación y su orden relativo dentro del collar.

\section{AGRADECIMIENTOS}

Este estudio se enmarca en el Proyecto I+D HAR2008-06477-C03-02, financiado por el Ministerio de Ciencia e Innovación y gracias al Contrato de investigación OT2008/0233 de la UGEA-PHAM en la Universidad de Cádiz. El ADIF financió íntegramente la excavación. Los autores agradecen a todos los que con su trabajo hicieron posible la excavación de Chousa Nova 1 y a Ignacio Jaime Senín, arqueólogo de la Xunta de Galicia, Departamento Territorial de Lugo, la 
imagen de la reconstrucción del enterramiento de la figura 6 .

\section{BIBLIOGRAFÍA}

Alonso Matthías, F. y Bello Diéguez, J. M. 1995: "Aportaciones del dolmen de Dombate al megalitismo noroccidental: dataciones de carbono 14 y su contexto arqueológico". 1. ${ }^{\circ}$ Congresso de Arqueologia Peninsular (Porto 1993) VII. Trabalhos de Antropologia e Etnologia 35 (3): 153-181.

Álvarez Fernández, E.; Peñalver, E. y Delclós, X. 2005: "La presencia de ámbar en los yacimientos prehistóricos (del Paleolítico Superior a la Edad del Bronce) de la Cornisa Cantábrica y sus fuentes de aprovisionamiento". Zephyrus 58: 159-182.

Anderson, K. B.; Winans, R. E. y Botto, R. E. 1992: "The nature and fate of natural resins in the geosphere -II Identification, classification and nomenclature of resinites". Organic Geochemistry 18: 829-841.

Arribas, A.; Galán, E.; Martín, J. M.; Nicolau, J. y Salvador, P. 1971: "Estudio mineralógico de la variscita de Palazuelo de las Cuevas". Stvdia Geológica Salmanticensia 2: 115-132.

Beck, C. W.; Wilbur, E.; Meret, S.; Kossove, D. y Kermani, 1965: "The infra-red spectra of amber and the identification of Baltic amber". Archaeometry 8: 96-109.

Domínguez-Bella, S. 2004: "Variscite, a prestige mineral in the Neolithic-Aeneolithic Europe. Raw material sources and possible distribution routes". Slovak Geological Magazine 1-2: 151-158.

Domínguez-Bella, S.; Álvarez Rodríguez, M. ${ }^{\mathrm{a}}$ A. y Ramos Muñoz, J. 2001: "Estudio analítico de las cuentas de collar de ámbar del dolmen de Alberite (Villamartín, Cádiz). Naturaleza química y mineralógica e implicaciones sobre su origen". En B. Gómez Tubío, M. A. Respaldiza y M. L. Pardo (eds.): III Congreso Nacional de Arqueometría (Sevilla 1999): 621-630. Sevilla.

Domínguez-Bella, S. y Morata Céspedes, D. 1995: "Aplicación de las técnicas mineralógicas y petrológicas a la arqueometría. Estudio de materiales del dolmen de Alberite (Villamartín, Cádiz)". Zephyrus XLVIII: 129-142.

Domínguez Bella, S.; Nieto, J. M.; Nocete, F.; Calvo Ramos Muñoz, J. y Sáez R. 2003: "ICP-MS en Arqueometria. Variscitas arqueológicas en el oeste peninsular, Origen de las materias primas geológicas". Boletín de la Sociedad Española de Mineralogía 26-A: 113-114.

Domínguez-Bella, S.; Ramos Muñoz, J.; Álvarez, M. A. y Forteza, M. 1998: "Neolithic amber. Mi- neralogical and chemical characterization of amber necklace beads from Alberite Dolmen. Villamartín, Cádiz, Spain”. Libro de Abstracts del World Congress on Amber Inclusions: 155. Vitoria.

Fábregas Valcarce, R. 1992: Megalitismo del Noroeste de la Península Ibérica. Tipología y secuencia de los materiales líticos. Universidad Nacional de Educación a Distancia. Madrid.

Fábregas Valcarce, R.; Bonilla Rodríguez, A. y César Vila, M. 2007: Monte dos Remedios (Moaña, Pontevedra). Un asentamiento de la prehistoria reciente. Tórculo. Santiago de Compostela.

Fábregas Valcarce, R. y Vilaseco Vázquez, X. I. 2006: "En torno al megalitismo gallego". En R. Fábregas Valcarce y F. Carrera Ramírez (eds.): Arte Parietal Megalítico en el Noroeste Peninsular. Conocimiento y conservación. Tórculo. Santiago de Compostela: 11-36.

Lima Oliveira, E. 2000: La Arqueología en la Gasificación de Galicia 12: intervenciones en yacimientos prehistóricos. Laboratorio de Arqueoloxía e Formas Culturais, Universidade de Santiago de Compostela. Santiago de Compostela.

Mañana Borrazás, P. 2005: "Túmulo 5 de Forno dos Mouros (Ortigueira, A Coruña). Primeiros resultados". Cuadernos de Estudios Gallegos LII: $39-79$.

Ramos, J. y Giles, F. (eds.) 1996: El dolmen de Alberite (Villamartín). Aportaciones a las formas económicas y sociales de las comunidades neolíticas en el Noroeste de Cádiz. Universidad de Cádiz y Ayuntamiento de Villamartín. Cádiz.

Reimer, P. J.; Baillie, M. G. L.; Bard, E.; Bayliss, A.; Beck, J. W.; Blackwell, P. G.; Buck, C. E.; Burr, G. S.; Cutler, K. B.; Damon, P. E.; Edwards, R. L.; Fairbanks, R. G.; Friedrich, M.; Guilderson, T. P.; Herring, C.; Hughen, K. A.; Kromer, B.; McCormac, F. G.; Manning, S. W.; Ramsey, C. B.; Reimer, P. J.; Reimer, R. W.; Remmele, S.; Southon, J. R.; Stuiver, M.; Talamo, S.; Taylor, F. W.; Van der Plicht, J. y Weyhenmeyer, C. E. 2004: "INTCAL04 terrestrial radiocarbon age calibration, 0-26 cal kyr BP". Radiocarbon 46-3: 1029-1058.

Savkevich, S. y Shaks, I. 1964: "Infrared absorption spectra of Baltic amber (Succinite)". Zhurnal Prikladnoi Chimii 37: 1120-1122.

Vaquero Lastres, J. 1999: Les extrêmes distincts. La configuration de l'espace dans les sociétés ayant batî des tertres funéraires dans le Nord-Ouest Iberíque. British Archaeological Reports International Series 821. Tempus Reparatum. Oxford.

Vijande, E. 2009: "El poblado de Campo de Hockey (San Fernando, Cádiz): resultados preliminares y líneas de investigación futuras para el conocimiento de las formaciones sociales tribales en la Bahía de Cádiz (tránsito V. ${ }^{\circ}$-IV. ${ }^{\circ}$ milenios a.n.e.)". Revista 
Atlántica-Mediterránea de Prehistoria y Arqueología Social XI: 265-284.

Vilaça, R.; Beck, C. W. y Shout, E. C. 2002: "Provenience analysis of prehistoric amber artifacts in Portugal". Madrider Mitteilungen 43: 61-74.

Vilaseco Vázquez, X. I. 1997/98: "Datos sobre a presenza de estruturas de acceso en túmulos mega- líticos da provincia de Lugo". Boletín do Museo Provincial de Lugo VIII (1): 125-156.

Vilaseco Vázquez, X. I. 2006: "Á beira da morte. Algúns exemplos europeos sobre a utilización do contorno inmediato aos monumentos megalíticos funerarios como posíbeis modelos para Galicia". Gallaecia 25: 7-44. 